\title{
A Dual-Parameter Fusion Distributed Optical Fiber Sensor System for Oil and Gas Pipeline Monitoring
}

\author{
Baoji Li, Hongliang Wu, Yangquan Xie, Zhiyong Dai \\ School of Optoelectronic Science and Engineering, University of Electronic Science and Technology of China, Chengdu, China \\ Email: emzydai@uestc.edu.cn
}

How to cite this paper: Li, B.J., Wu, H.L., Xie, Y.Q. and Dai, Z.Y. (2019) A Dual-Parameter Fusion Distributed Optical Fiber Sensor System for Oil and Gas Pipeline Monitoring. Journal of Applied Mathematics and Physics, 7, 2909-2916. https://doi.org/10.4236/jamp.2019.711200

Received: November 15, 2019 Accepted: November 24, 2019 Published: November 27, 2019

\begin{abstract}
For oil and gas pipeline monitoring applications, this paper proposed a dual-parameter fusion distributed fiber optic sensor system that enables distributed temperature and distributed vibration measurements in a single fiber. Through the fiber-scattering spectrum time domain detection combined with coded pulse sequence and Raman scattering spectrum is obtained, which realizes high-resolution temperature measurement and wide-band vibrational wave measurement. The experimental results show that, on $10 \mathrm{~km}$ optical fiber measurement, temperature resolution up to $0.1^{\circ} \mathrm{C}$ and vibration response frequency range $20 \mathrm{~Hz}-5 \mathrm{kHz}$. This sensing system achieves temperature and vibration dual-parameter measurements with fiber optics, greatly simplifying the system and facilitating installation and it can be widely used in oil and gas pipeline monitoring.
\end{abstract}

\section{Keywords}

Oil and Gas Pipeline Monitoring, Leakage Monitoring, Dual-Parameter, Optical Fiber Sensor, CCPONS Code

\section{Introduction}

Oil and gas pipelines can easily lead to pipeline leakage due to geological disasters, man-made damage and corrosion of pipelines themselves. At present, commonly used pipeline monitoring methods include: ultrasonic method, negative pressure wave method, pressure gradient method, and the like. However, these methods are not ideal in practical applications, can only be used for pipeline leakage alarm and cannot be used for early warning monitoring [1].

The distributed optical fiber sensor has the characteristics of long measuring 
distance and anti-electromagnetic interference. It is very suitable for oil and gas pipeline leakage monitoring. The distributed optical fiber temperature sensors (DTS) and distributed optical fiber vibration sensors (DVS) were also been verified by preliminary engineering application in oil and gas pipeline leakage monitoring [2] [3] [4] [5]. If a leak in the oil and gas pipeline, the temperature will change at the leak. The distributed fiber temperature sensor can detect the temperature change to realize the leak alarm detection. Shown high stability and low false alarm performance, but need long response time to leakage events [6]. The distributed optical fiber vibration sensors were used to monitor the oil and gas pipelines, which has the advantages of fast response speed, third-party intrusion early warning monitoring, etc., but is easily interfered by the external environment, prone to false alarm [7].

This paper proposes a dual-parameter fusion distributed optical fiber sensing system that combines fiber-scattered spectral time domain detection with pulsed coded sequence detection to achieve high signal-to-noise ratio acquisition of Rayleigh scattering and Raman scattering simultaneously. High-precision distributed temperature measurement and high-sensitivity distributed vibration disturbance measurement was realized. The fusion of the two parameters of temperature and vibration can overcome the shortcomings of the two parameters and improve the reliability of oil and gas pipeline safety monitoring.

\section{Technical Principle}

\subsection{The Principle of Coding Time Domain Detection Distributed Optical Fiber Sensor}

In order to realize wide-band detection, the distributed optical fiber vibration sensor cannot perform the averaging processing of the backscattered light. It is necessary to increase the signal-to-noise ratio of the scattered signal by increasing the peak power of the detected pulse light; however, the narrow linewidth light source used in the system is easier. Producing a non-linear effect that is unfavorable to the test requires controlling the peak power of the optical pulse to a low level. In order to solve this contradiction, pulse coding technology can increase the total energy of the injected fiber and increase the intensity of the scattered light signal by increasing the number of emitted light pulses without increasing the peak power of the optical pulse.

Up to now, the coding methods used in engineering practice mainly include Simplex code, Golay code and Complementary Correlated Prometheus Orthonormal Sequence (CCPONS), as shown in Figure 1, comparison of coding gains of three different coding modes. In this paper, we choose CCPONS, because CCPONS can improve the SNR most.

The block diagram of the dual-parameter fusion fiber optic sensing system is shown in Figure 2. The entire sensing system consists of two parts: DVS and DTS. Among them, NLL (narrow linewidth laser), AOM (acoustic light modulator), EDFA1 (doped fiber amplifier), circulator 1 and detector 1 belong to DVS part; 


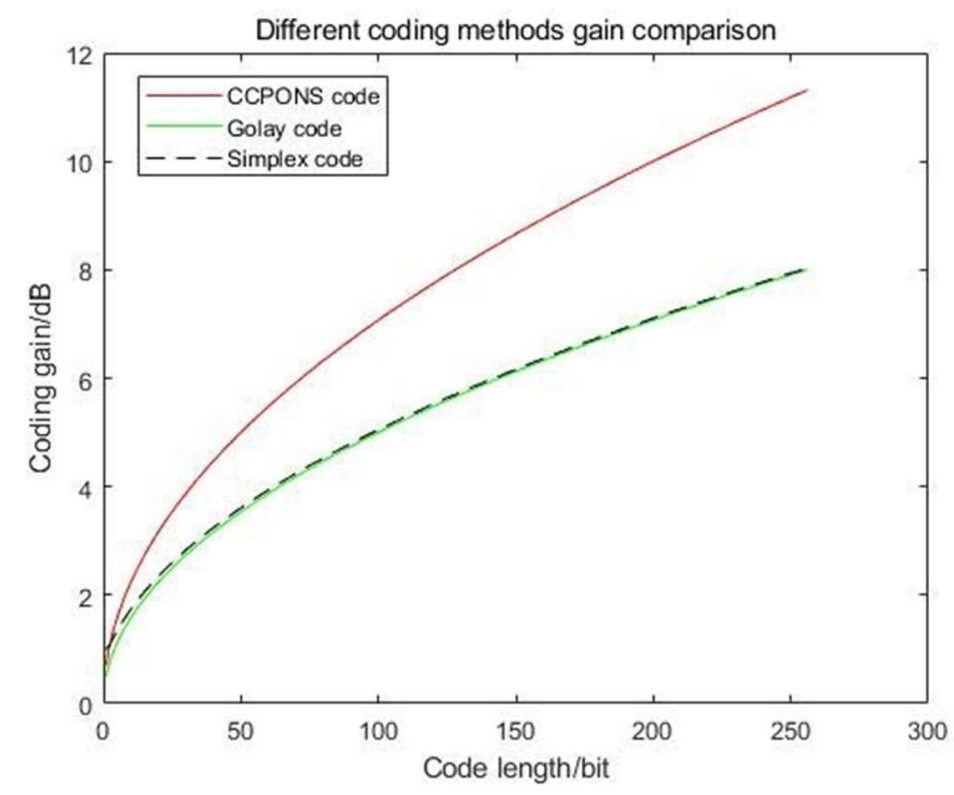

Figure 1. Comparison of coding gains of three different coding modes.

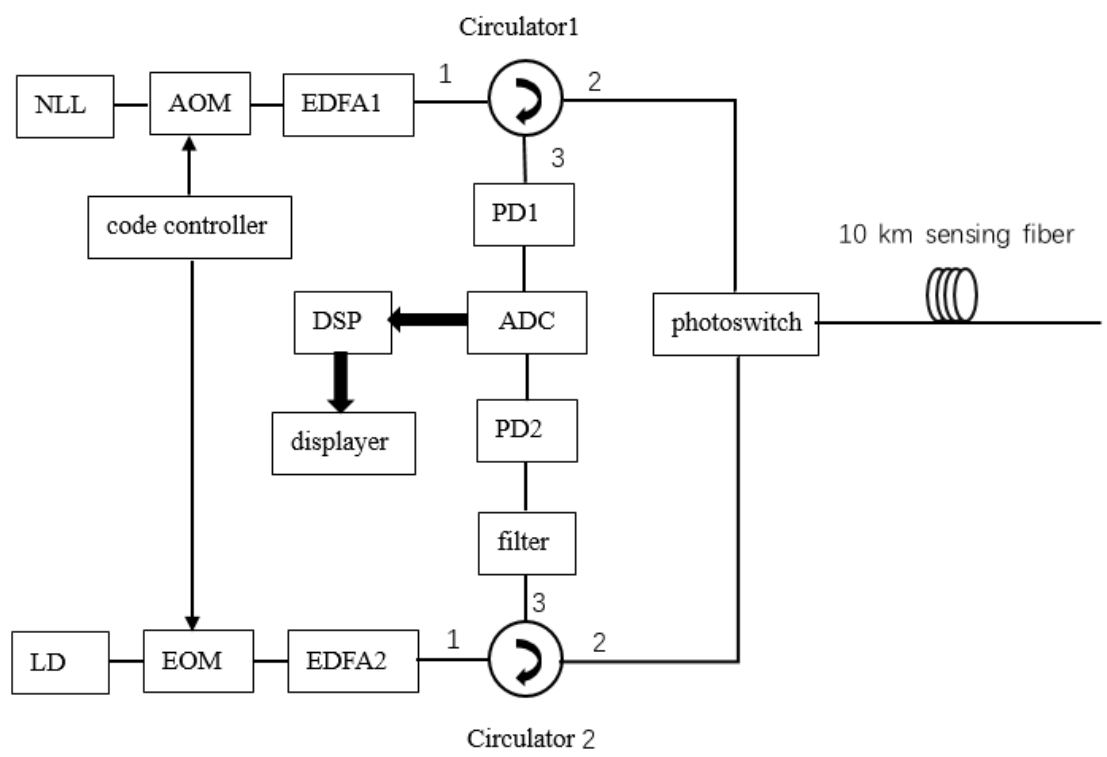

Figure 2. Block diagram of a dual-parameter fusion fiber optic sensing system.

LD (laser), EOM (electro-optic modulator) EDFA2 (doped fiber amplifier), circulator 2, fiber filter and detector 2 belong to the DTS part.

Here, the power of narrow linewidth laser is $10 \mathrm{~mW}$, the line width is $1 \mathrm{kHz}$; the detector bandwidth is $50 \mathrm{MHz}$; the data acquisition card sampling rate is 100 $\mathrm{MS} / \mathrm{s}$, the sampling precision is $16 \mathrm{bit}$; the optical pulse generated by the photoelectric modulator has a minimum pulse width of $10 \mathrm{~ns}$.

When sensing system is working, the DVS and the DTS are time-division multiplexed by the photoswitch, the sensing fiber was alternately connected to the DVS or DTS, complete distributed measurement of vibration and temperature. 


\subsection{CCPONS Pulse Code Distributed Optical Fiber Temperature Sensor}

The distributed temperature measurement can be realized by the extraction and detection of Raman scattering light signal. The system composition is shown in the part of ROTOR (lower part) in Figure 2. The anti Stokes light is separated by optical filtering for photoelectric detection. Assuming that the peak power of the optical pulse in the injected fiber is $P_{0}$ and the spontaneous Raman scattering occurs at the distance $z$, the optical power of the backward Raman scattering signal obtained at the injection end of the optical pulse is:

$$
P_{A S}[Z, T(Z)]=P_{0} v_{g} t \Gamma_{A S} \rho_{A S} 10^{-\left[\alpha\left(\lambda_{0}\right)+\alpha\left(\lambda_{A S}\right)\right] Z / 10}
$$

where, $P_{A S}[Z, T(Z)]$ is the light power of anti Stokes signal, $Z$ is the coordinate of scattering point, $T(Z)$ is the temperature value at coordinate $Z, v_{g}$ is the group velocity of pulse propagation, $\tau$ is the light pulse width, $\alpha\left(\lambda_{0}\right)$, $\alpha\left(\lambda_{A S}\right)$ is the attenuation coefficient of incident light, Stokes light and anti Stokes light in optical fiber respectively, $\lambda_{0}, \lambda_{A s}$ is the backscattering coefficient of Stokes light and anti Stokes light respectively, $\rho_{A S}$ is the population number of the lower and higher energy level of optical fiber molecule, which is related to temperature, can be expressed as:

$$
P_{A S}=\frac{\exp [-h \Delta v / K T(Z)]}{1-\exp [-h \Delta v / K T(Z)]}
$$

where, $\Delta v$ is the molecular vibration frequency in the optical fiber, $h$ is Planck constant, $K$ is Boltzman constant. The traditional distributed light temperature sensor uses the two-way demodulation of Stokes light and anti Stokes light. Combined with the digital processing method, formula (1) can realize the single optical path demodulation. Single optical path demodulation can significantly eliminate the nonlinear effect and ensure long-distance and high-resolution temperature measurement.

When the high-pressure gas in the natural gas pipeline leaks, the temperature of the objects around the leakage hole will drop rapidly. By monitoring the abnormal temperature changes along the natural gas pipeline using the communication optical cable, the leakage event on the gas transmission pipeline can be detected and the location of the leakage event can be accurately located.

\subsection{CCPONS Pulse Coded Distributed Fiber Optic Vibration Sensor}

The structure of the pulse coded distributed fiber-optic vibration sensor is shown as DVS part in Figure 2 (upper part). When natural gas leaks, the natural gas will friction with the leakage wall, which induce stress waves on the pipe wall to make pipe vibrate. The vibration generated by the pipe will act on the fiber through the surrounding medium, changing the length and refractive index of the fiber. The phase of the light propagating in the fiber is modulated, and its expression is

$$
\varphi_{s}=\beta \Delta L+L \Delta \beta
$$


where $\varphi_{s}$ is the phase change amplitude of the light wave, $\beta$ is the propagation constant of the light wave in the fiber; $L$ is the length of the fiber affected by the pipe leakage noise. The leakage signal acted on the sensing fiber modulate the phase of the light propagating in the fiber, the phase change by the leakage signal is

$$
\delta_{\varphi}=\varphi_{s} \sin \omega_{s} t
$$

where $\varphi_{s}$ is defined as the magnitude of the phase change after modulation, and $\omega_{s}$ is the frequency of the leakage signal. The optical phase change caused by the leakage signal is:

$$
\Delta \theta(t)=\theta_{1}(t)-\theta_{2}(t)=2 \varphi_{s} \cos \omega_{s}\left(t-\frac{\tau_{1}+\tau_{2}}{2}\right) \sin \omega_{s}\left(\frac{\tau_{2}-\tau_{1}}{2}\right)
$$

Let $\tau_{s}=\frac{\tau_{2}-\tau_{1}}{2}-\frac{n s}{c}$, where $c$ is the speed of light in the air, $n$ is the refractive index of the fiber, and $s$ is the distance between the leak point and the midpoint of the interferometer. Let $\tau_{T}=\tau_{1}+\tau_{2}=\frac{L n}{c}$, where $\tau_{T}$ denotes the total time that the light passes through the fiber, and $L$ is the length of the fiber. Rewrite formula (6) as follows

$$
\Delta \theta(t)=\theta_{1}(t)-\theta_{2}(t)=2 \varphi_{s} \cos \omega_{s}\left(t-\frac{\tau_{T}}{2}\right) \sin \omega_{s} \tau_{s}
$$

By demodulating $\Delta \theta(t)$ through signal demodulation technology, the soil vibration signal caused by gas leakage can be detected. The spectral information of the vibration signal can be obtained by FFT operation on the vibration signal. When the distributed optical fiber vibration sensor detects an abnormal vibration signal, compared with the temperature change at the same position, if the temperature module detects an abnormal temperature change, it can be confirmed that a leak occurs at the position.

\section{Experimental Results and Discussion}

A simulated leak test was carried out with a communication cable laid in the same trench on a natural gas pipeline. The length of the communication optical cable was $8086 \mathrm{~m}$, and the test was performed at $7800 \mathrm{~m}$. A high-pressure pipe was connected from the high-pressure gas cylinder, and the high-pressure pipe outlet was fixed vertically at a distance of $20 \mathrm{~cm}$ from the communication optical cable. The air outlet hole and the communication optical cable were at the same horizontal plane and buried at a depth of $1.5 \mathrm{~m}$ underground. A chemically stable and non-toxic nitrogen gas was used to simulate natural gas leakage with a nitrogen pressure of $2 \mathrm{MPa}$. When the nitrogen gas began to leak, the DVS module could immediately detect the vibration signal generated by the gas leakage, DTS module could detect the abnormal decrease of the temperature in the vibration position. The temperature changes detected by the temperature sensing module and the vibration signals detected by the vibration sensing module are shown in Figure 3 respectively. 


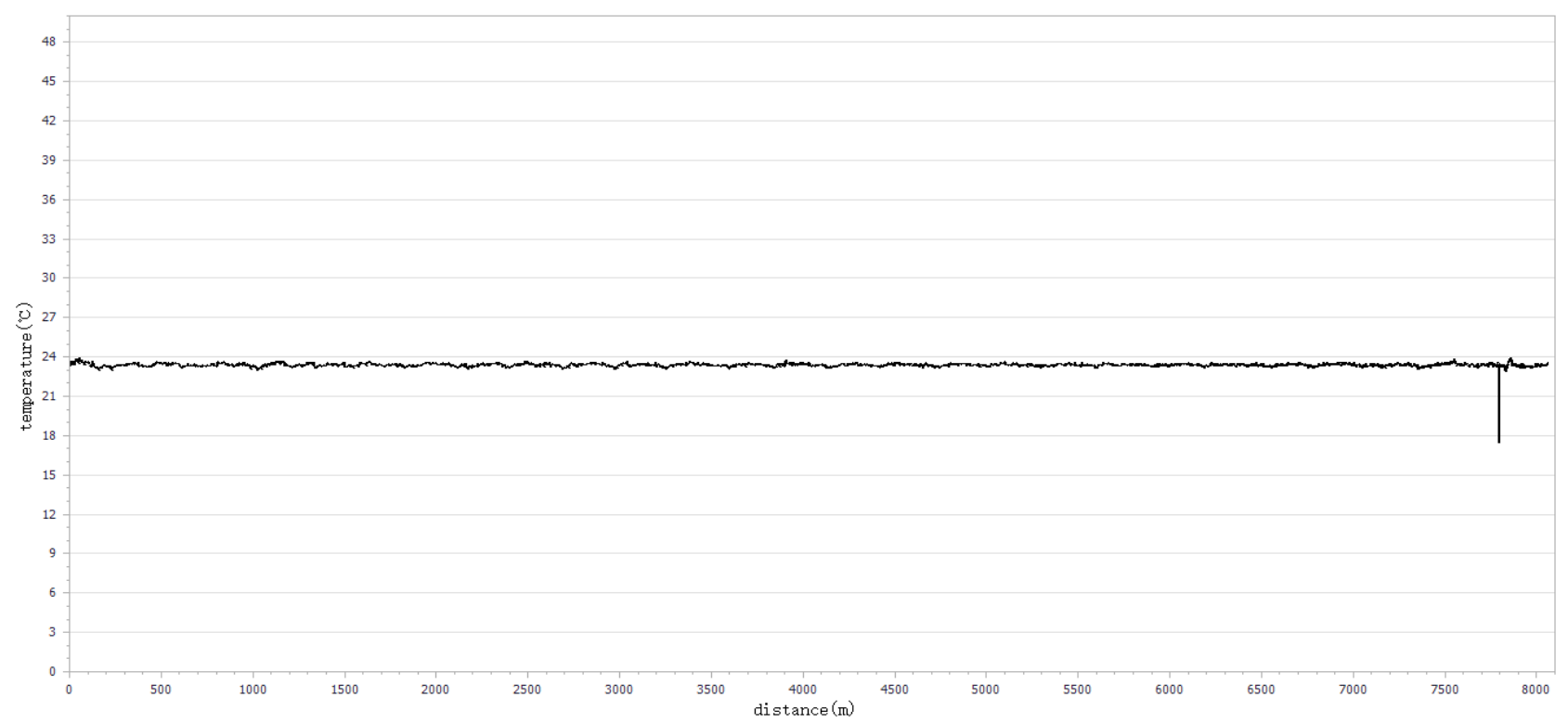

(a)

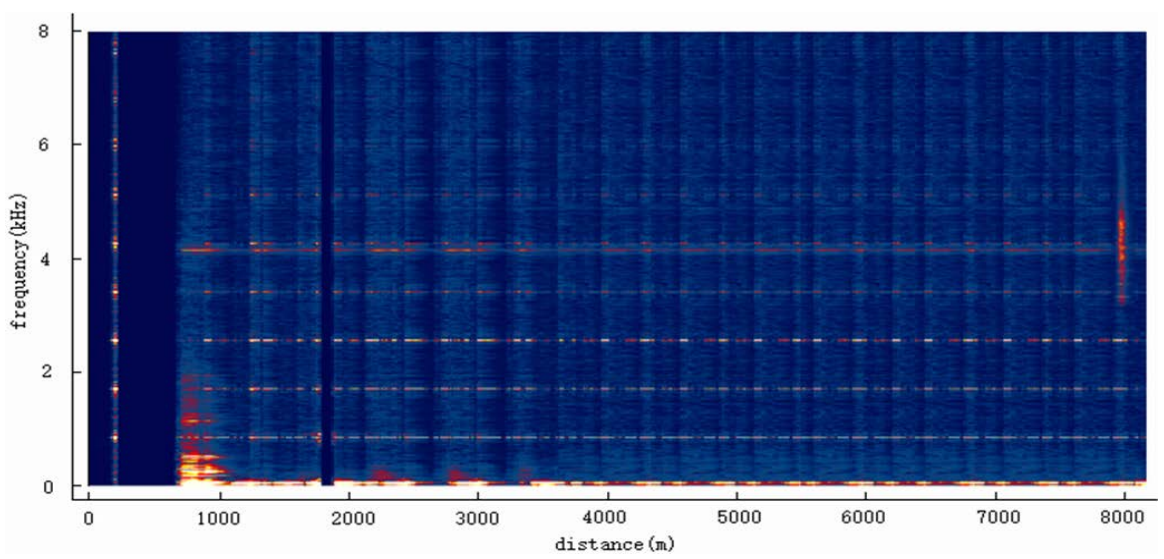

(b)

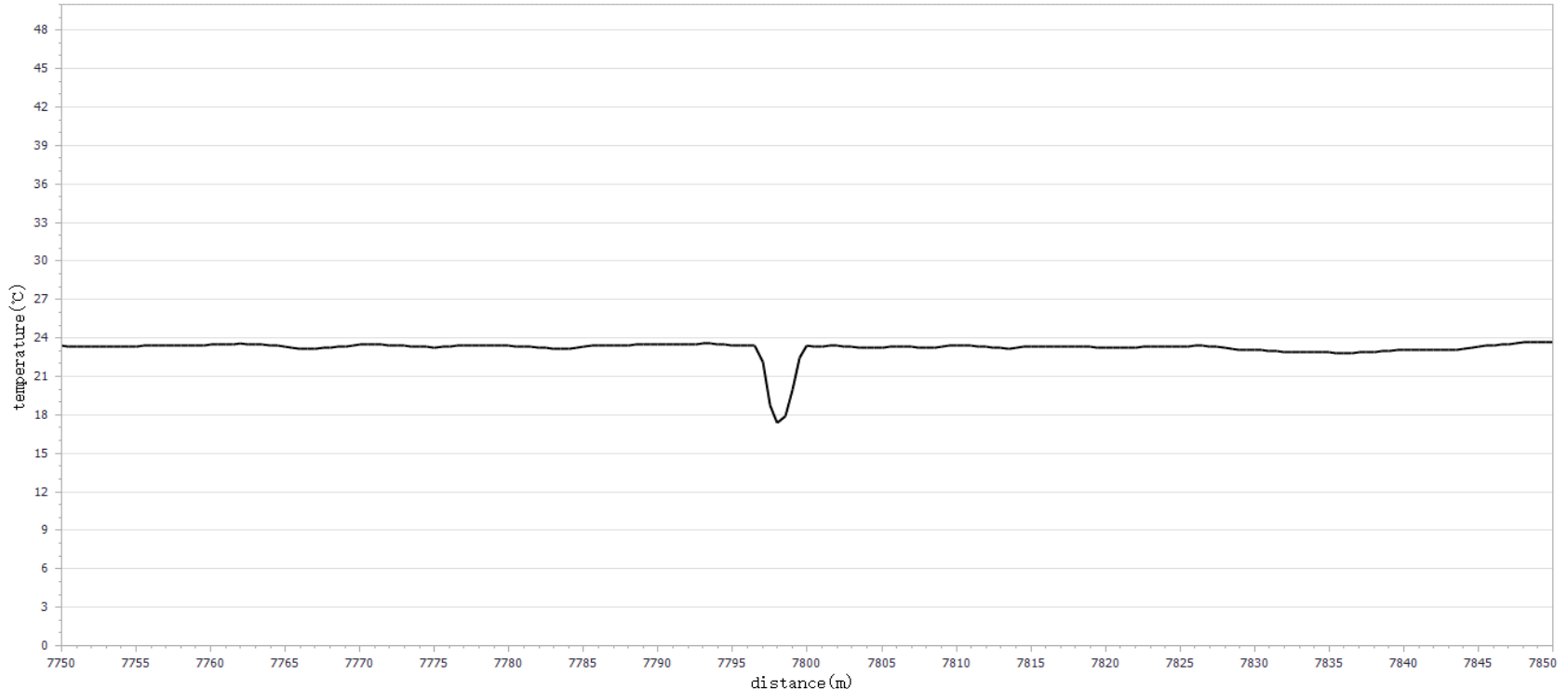

(c) 


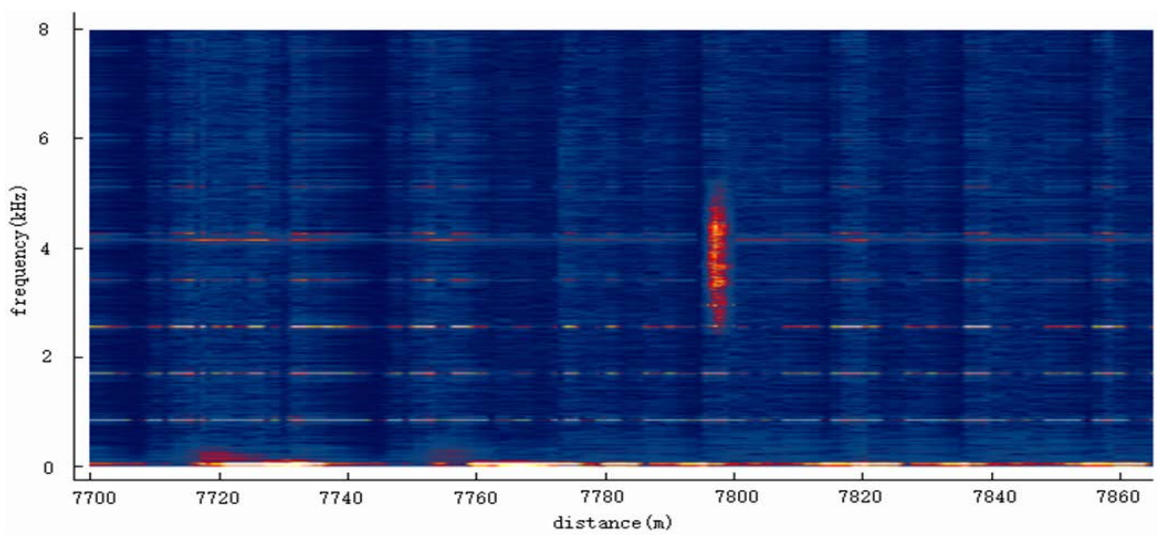

(d)

Figure 3. Test results of simulated leakage measured by Dual-parameter distributed fiber optic sensor system. (a) Temperature signal on the whole optical cable; (b) Vibration spectrum signal on the whole optical cable; (c) Temperature change of leakage position; (d) Vibration spectrum signal of leakage position.

The experiment results showed that the DTS detected an abnormal decrease of the temperature at $7798.5 \mathrm{~m}$, and the temperature dropped by $6.03^{\circ} \mathrm{C}$ at most. The DVS module detected an abnormal vibration signal at $7796 \mathrm{~m}$, and the vibration signal frequency is concentrated in the range of $4 \mathrm{kHz}$ to $4.5 \mathrm{kHz}$. It is clear that the DTS module and the DVS module are both detect the abnormal signal generated by leakage. The leakage positions detected by the two sensing modules are consistent, and the positioning error is less than $4 \mathrm{~m}$.

\section{Conclusion}

In this paper we proposed a dual-parameter distributed optical fiber sensing system that combines fiber-optic scattering spectrum time-domain detection with pulsed coded sequence detection to achieve high signal-to-noise ratio (SNR) acquisition of Rayleigh scattered light and Raman scattered light with one single fiber. The experimental results show that the measurement distance is 10 $\mathrm{km}$, the temperature resolution is $0.1^{\circ} \mathrm{C}$, and the vibration response frequency range is $20 \mathrm{~Hz}-5 \mathrm{kHz}$. The system combines two parameters measurement functions onto the same fiber, simplifying the test system, facilitating the application and installation, and reducing monitoring costs. In summary, the sensor system proposed in this paper has obvious technical advantages compared with other traditional leak monitoring systems, and it has great application potential in the field of oil and gas pipeline leakage monitoring.

\section{Acknowledgements}

This project was supported by The National Basic Research Program of China (No. 2016ZX05021-005-010HZ).

\section{Conflicts of Interest}

The authors declare no conflicts of interest regarding the publication of this paper. 


\section{References}

[1] He, B. (2017) Research on Application of Distributed Fiber Sensing Technique in Process of Monitoring Oil and Gas Pipelines. Dalian University of Technology, Dalian.

[2] Xi, T.T. (2018) Application of Distributed Optical Fiber Temperature Sensor in High Temperature Logging of Oil Field. Petrochemical Industry Technology, 25, 16-17.

[3] Xu, C., Yan, J.Y., Mao, T.Q., et al. (2017) Research Progress of Distributed Raman Fiber Sensing Technology. Journal of Functional Materials and Devices, 23, 73-76.

[4] Wang, S.S. (2012) Application of Temperature Logging in Oil and Gas Field Development. China Petroleum and Chemical Standard and Quality, 32, 193.

[5] Kuang, X., Wu, H.T., Zhang, J.D., et al. (2018) Research Progress of Distributed Multi-Parameter Fiber Sensing Technology. Opto-Electronic Engineering, 45, 66-81.

[6] He, X.L. (2012) High Precision Optical Fiber Sensing System and Its Application in Oilfield Logging. Dalian University of Technology, Dalian.

[7] Zhao, L. (2014) Analysis on the Development Prospect of China's Optical Fiber Sensor Market. Study on Optical Communications, 2014, 45-48. 